\title{
NOUVELLE
}

\section{Identification de cibles thérapeutiques et repositionnement de médicaments par analyses de réseaux géniques}

Andrée Delahaye-Duriez ${ }^{1-3}$, Clémence Réda ${ }^{1,4}$, Pierre Gressens ${ }^{1}$
${ }^{1}$ NeuroDiderot, UMR 1141, Inserm, Université de Paris, Sorbonne Paris Cité, Hôpital Robert Debré, 48, boulevard Sérurier, 75019 Paris, France.

${ }^{2}$ Université Paris 13, Sorbonne Paris Cité, UFR de santé, médecine et biologie humaine, 93000 Bobigny, France. ${ }^{3}$ Service histologie-embryologie-cytogénétique-biologie de la reproduction-CECOS, Hôpital Jean Verdier, AP-HP, 93140 Bondy, France.

${ }^{4}$ École Normale Supérieure Paris-Saclay, 94230 Cachan, France.

andree.delahaye@inserm.fr

\section{Développement et validation de thérapies}

Malgré les avancées technologiques et l'amélioration des connaissances sur les maladies humaines, les progrès thérapeutiques restent lents. L'identification et la validation de cibles thérapeutiques demeurent des étapes critiques du processus de découverte de médicaments. Le développement de nouvelles molécules est long et coûteux. La validation de l'efficacité des molécules candidates et les démarches nécessaires pour obtenir une mise sur le marché représentent des investissements importants pour l'industrie pharmaceutique. Une alternative efficace est le repositionnement de médicaments ou drug repurposing. Le repositionnement de médicament consiste à utiliser des molécules déjà présentes sur le marché pour les appliquer à d'autres maladies qui ne relèvent pas de l'indication médicale d'origine [1] $(\rightarrow)$.

$(\rightarrow)$ Voir l'Éditorial de C. Martinat et M. Peschanski, $m / s n^{\circ} 12$, décembre 2018, page 1019

\section{Identification des réseaux}

de régulation génique perturbés

en pathologie

La caractérisation d'une maladie en termes de perturbations de réseaux de régulation génique, ou GRN (gene regulatory network) offre des opportunités considérables pour accélérer l'identification de cibles thérapeutiques et guider le repositionnement de médicaments. Les GRN sont des réseaux complexes de

gènes, de protéines, et de petites molécules dont les interactions contrôlent les fonctions biologiques. Grâce aux efforts concertés de la génétique, de la biologie moléculaire, de la biochimie et de la physiologie, des quantités énormes de données portant sur les différents composants de ces réseaux sont maintenant disponibles. Les domaines de la génomique fonctionnelle et de la biologie des systèmes développent et utilisent des modèles mathématiques afin d'étudier et de prédire le comportement de ces GRN. Une des approches pour les identifier est d'étudier la co-expression des gènes. Si l'on dispose de suffisamment de profils d'expression recueillis à partir de plusieurs échantillons et selon plusieurs conditions expérimentales, on peut alors évaluer la similarité de l'expression de chaque paire de gènes et construire un réseau génique à partir duquel il sera possible de regrouper les gènes présentant un profil d'expression similaire et ainsi construire des modules de gènes co-exprimés (Figure 1). Ces modules sont intéressants sur le plan biologique. Ils sont en effet fonctionnellement liés et codent des protéines qui, pour la plupart, interagissent entre elles. Ils reflètent également les GRN en révélant leur contrôle et les liens de régulation qui les régissent [2]. L'intégration avec des données de susceptibilité génétique ou des informations phénotypiques permet de connecter des modules de gènes co-exprimés ou des GRN à des états pathologiques. Ainsi, on peut identifier des voies de transmission de signaux cellulaires impliqués dans une maladie et leurs régulateurs comme de nouvelles cibles thérapeutiques [3]. II existe plusieurs exemples fructueux dans lesquels l'application d'approches de cartographie d'expression de traits quantitatifs d'expression (expression quantitative trait loci, eQTL) à des modules de gènes co-exprimés associés à une maladie a permis l'identification de régulateurs clés comme celle de possibles cibles thérapeutiques [4-6]. Pourtant, les régulateurs identifiés ne constituent pas toujours des cibles facilement exploitables d'un point de vue thérapeutique. C'est pourquoi d'autres approches intègrent les régulations liées spécifiquement aux protéine kinases, comme X2K (programme eXpression $2 k i-$ nase) [7] ou aux récepteurs membranaires [8].

\section{Exemple d'identification d'un} récepteur membranaire comme cible thérapeutique

Dans un travail interdisciplinaire coordonné par Michael Johnson et Enrico Petretto, en collaboration avec UCB Pharma et Clarivates Analytics, une série d'analyses appelée CRAFT (causal reasoning analytical framework for target discovery) a été développée de façon à sélectionner les récepteurs membranaires participant à la régulation de l'expression génique dans la direction opposée à celle observée en pathologie [8]. Comme preuve de concept, CRAFT 
A

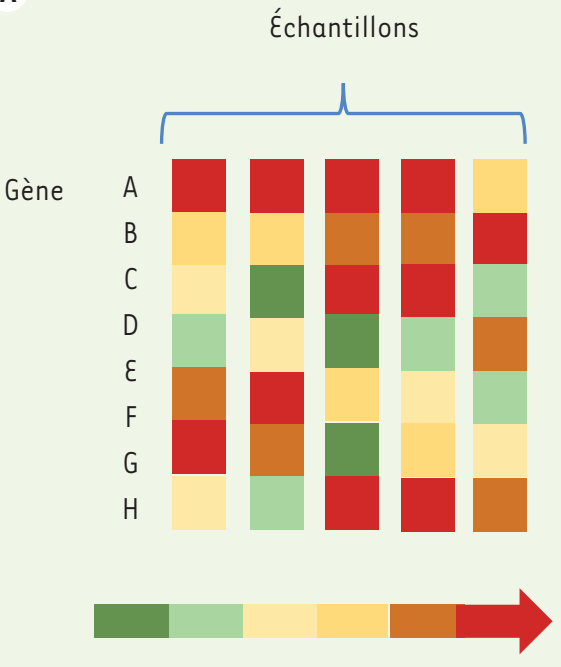

Niveau d'expression de gène

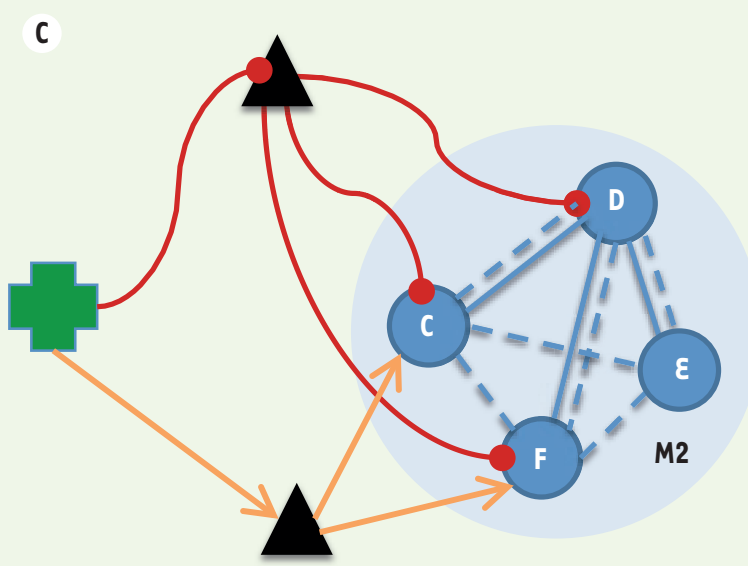

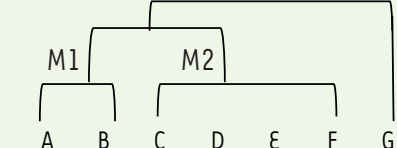
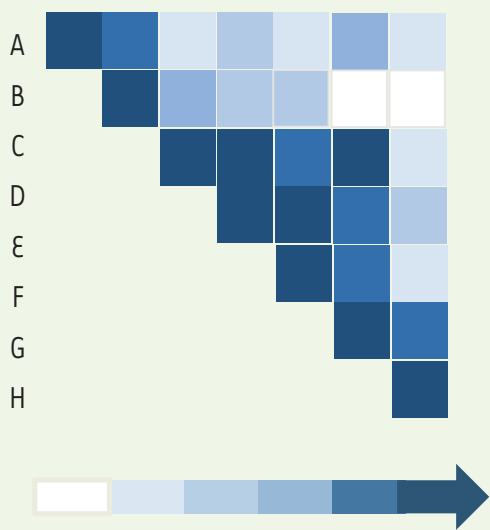

Coefficient de similarité

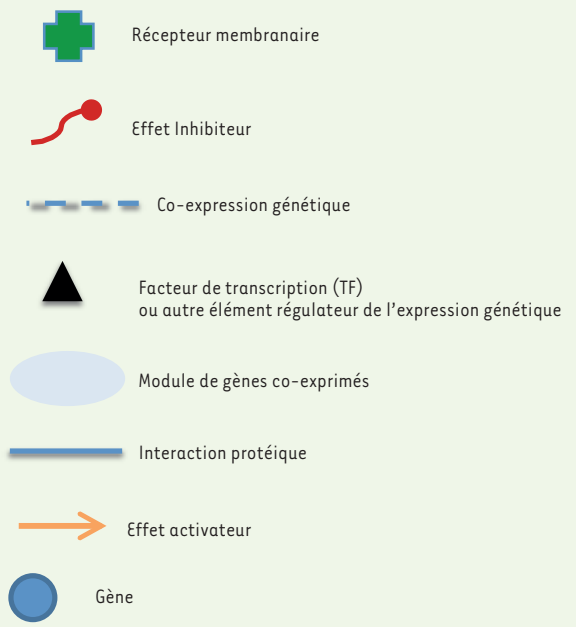

Figure 1. Du profil d'expression au réseau de régulation génique. A. Les profils d'expression pour chaque gène considéré (ici, on considère 7 gènes notés $A, B, C, D, \varepsilon, F$ et $G$ ) sont construits à partir de données transcriptomiques issues de différents échantillons, en concaténant les niveaux d'expression du gène considéré pour chaque condition testée. $B$. On peut inférer les modules de coexpression de gènes en calculant la distance entre les profils d'expression de chaque paire de gènes d'intérêt et en appliquant une méthode de classification non supervisée afin de les grouper en modules de gènes ayant des niveaux d'expression similaires sous les mêmes conditions (ici, deux modules sont inférés, $\mathrm{Ml}$ comportant les gènes $A$ et $B$, et $M 2$ contenant les gènes $C, D, \varepsilon$ et $F$ ). $C$. Réseau de régulation génique ou GRN (gene regulatory network) considérant les éléments régulateurs de l'expression des gènes du module $M 2$, par exemple, les facteurs de transcription (TF), et un récepteur membranaire. Les interactions de régulation (activatrices ou inhibitrices de l'expression) sont soit inférées par corrélation entre les niveaux d'expression, soit prouvées expérimentalement (et une relation causale est alors établie).

a été appliqué à l'épilepsie et a permis d'identifier CSFIR (colony-stimulating factor 1 receptor) comme une cible thérapeutique. Dans un modèle murin d'épilepsie du lobe temporal (TLE, temporal lobe epilepsy) induite par l'injection de pilocarpine, les réseaux de transcription dans les hippocampes ont été analysés et des modules de gènes co-exprimés associés à la maladie épileptique ont été isolés. Douze modules se sont révélés être différemment co-exprimés de manière significative entre des hippocampes épileptiques et des hippocampes témoins
(100 échantillons dans les deux cas). Pour hiérarchiser davantage les modules en fonction de leur relation à l'épilepsie, la corrélation entre l'expression de chaque module et la fréquence des crises a été explorée. Parmi les 9 modules dont l'expression était reliée aux crises, 
le module 18 s'est révélé être le plus significativement corrélé aux convulsions. Ce module était particulièrement enrichi en gènes connus pour coder des protéines impliquées dans les processus inflammatoires ainsi qu'en gènes marqueurs des cellules microgliales. Sept modules, dont ce module 18, sont conservés chez l'homme. Ils sont également co-exprimés de manière significativement différente dans les pièces chirurgicales d'hippocampes de patients atteints d'épilepsie du lobe temporal par rapport aux données obtenues à partir de prélèvements post-mortem sur des hippocampes d'individus dépourvus d'antécédent psychiatrique ou neurologique. La suite d'analyses CRAFT a été appliquée aux 7 modules candidats sélectionnés. CRAFT permet de modéliser les différents scénarios de régulation induite par des récepteurs membranaires sur des facteurs de transcription et leurs gènes cibles. Des tests statistiques ont permis d'ordonner ces récepteurs membranaires selon leur influence sur les gènes du module testé, dans une direction opposée à celle observée dans les hippocampes épileptiques. CSFIR (colony-stimulating factor 1 receptor) a été ainsi prédit comme un régulateur de deux des sept modules candidats (dont le module 18). Il a alors été émis I'hypothèse que le blocage de ce récepteur pourrait être une cible thérapeutique dans le traitement de l'épilepsie. La disponibilité du PLX3397 (Pexidartinib), un inhibiteur connu de CSFlR, a permis de tester expérimentalement cette hypothèse. Le blocage de CSFIR a atténué les crises épileptiques dans trois modèles précliniques d'épilepsie, ce qui a permis de confirmer qu'il était une cible thérapeutique potentielle pour lutter contre l'épilepsie acquise. CSFIR est un récepteur membranaire exprimé par les cellules de la lignée myéloïde, notamment les monocytes, les macrophages et la microglie. II a été suggéré que la survie des cellules microgliales dépendait de la signalisation induite par CSFIR, un traitement prolongé à fortes doses de PLX3397 (c'est-à-dire 5 à 6 fois supérieure à celle utilisée dans le travail de Srivastava et al. [8]) entraînant une réduction du nombre de cellules microgliales dans le cerveau de souris naïves [9]. Aux doses faibles de PLX3397, telles qu'utilisées par Srivastava et al. [8], il n'y a pas de mort cellulaire des cellules microgliales, mais les changements d'expression des gènes du module 18 associés à l'épilepsie sont inversés. L'efficacité antiépileptique de PLX3397 a été démontrée dans deux modèles murins d'épilepsie du lobe temporal : le modèle utilisant la pilocarpine et celui impliquant le kainate ${ }^{1}$. Dans les deux modèles, PLX3397 a significativement réduit la fréquence ou la durée des crises spontanées. En revanche, PLX3397 n'a pas montré d'efficacité dans trois modèles de crises aiguës chez les souris non épileptiques: le modèle de crise par électrochoc maximal, le modèle de crise à $6 \mathrm{~Hz}^{2}$ et le modèle au PTZ (pentylènetétrazole) ${ }^{3}$. Ces données distinguent clairement le PLX3397 de tous les médicaments antiépileptiques/ anticonvulsivants actuellement utilisés, qui sont généralement efficaces dans de tels modèles de crises épileptiques. Cette étude démontre donc que la restauration de l'expression des modules liés à la maladie vers un état sain permet de prédire l'efficacité thérapeutique et ouvre le pas à une accélération de l'identification et de la validation de nouvelles cibles thérapeutiques non seulement pour le traitement de l'épilepsie, mais également pour celui d'autres maladies.

\section{Application pour le repositionnement de médicaments}

La quantification de l'inversion des changements d'expression génique associés à un état pathologique peut être exploitée pour prédire l'efficacité

\footnotetext{
${ }^{1}$ Deux agents convulsivants qui induisent une épilepsie chronique.

${ }^{2}$ Correspondant à des décharges de pointe-ondes de $6 \mathrm{~Hz}$.

${ }^{3}$ Le pentylènetétrazole (PTZ) est un agent pharmacologique convulsivant.
}

des médicaments. Les composés thérapeutiques sont alors jugés non pas par leur affinité de liaison avec une protéine particulière, mais par leur capacité à induire une réponse transcriptionnelle anti-corrélée au programme de régulation de l'expression génique qui sous-tend l'état pathologique ou l'exposition à un facteur de risque. Les approches fondées sur ce paradigme de l'inversion de signature bénéficient de bases de données publiques de profils d'expression des gènes de lignées cellulaires traitées avec des composés chimiques, comme CMap (connectivity map) [10]. Par exemple, parmi les nombreux médicaments référencés dans cette base de données, l'acide valproïque, un médicament antiépileptique largement utilisé, a été prédit comme le meilleur pour inverser la signature d'expression génique d'un module associé à l'épilepsie [11]. Dans la continuité de CMap, la base de données LINCS (library of integrated network-based cellular signatures, http://www.lincsproject.org) des NIH (National institutes of health, États-Unis) s'enrichit considérablement et contient aujourd'hui des profils d'expression pour plus de 20000 molécules $[12,13]$. Ces données en accès libre représentent une énorme ressource pour effectuer des criblages in silico de molécules. La création de méthodes d'exploitation systématique de ces données pour générer de nouvelles hypothèses d'efficacité de médicaments est un défi qui nécessite la collaboration de chercheurs spécialisés en mathématiques, en informatique et en biologie. Ces recherches préparent la médecine de précision de demain qui aura, en plus, le défi d'intégrer les données individuelles des patients pour adapter leur traitement de façon personnalisée. $\diamond$

Identification of therapeutic targets and drug repurposing via gene network analysis

\section{LIENS D'INTÉRÊT}

Les auteurs déclarent n'avoir aucun lien d'intérêt concernant les données publiées dans cet article. 


\section{RÉFÉRENCES}

1. Martinat C, Peschanski M. L'heure est venue du (re) positionnement thérapeutique des maladies ultrarares. Med Sci (Paris) 2018 ; 34 : 1019-21.

2. Weirauch MT. Gene coexpression networks for the analysis of DNA microarray data. In : Dehmer M, Emmert-Streib F, Salvador A, Graber A, eds. Applied statistics for network biology: methods in systems biology. New York : Wiley, 2011 : 215.

3. Califano A, Butte AJ, Friend S, et al. Leveraging models of cell regulation and GWAS data in integrative network-based association studies. Nat Genet 2012; $44: 841-7$

4. Heinig M, Petretto $\varepsilon$, Wallace $C$, et al. A trans-acting locus regulates an anti-viral expression network and type 1 diabetes risk. Nature $2010 ; 467: 460-4$.
5. Kang H, Kerloc'h A, Rotival M, et al. Kcnn4 is a regulator of macrophage multinucleation in bone homeostasis and inflammatory disease. Cell Rep $2014 ; 8: 1210-4$

6. Johnson MR, Behmoaras J, Bottolo L, et al. Systems genetics identifies Sestrin 3 as a regulator of a proconvulsant gene network in human epileptic hippocampus. Nat Commun $2015 ; 6: 6031$.

7. Clarke DJB, Kuleshov MV, Schilder BM, et al. eXpression2Kinases (X2K) web: linking expression signatures to upstream cell signaling networks. Nucleic Acids Res 2018; 46 : W171-9.

8. Srivastava PK, van Eyll J, Godard P, et al. A systems-leve framework for drug discovery identifies CsflR as an anti-epileptic drug target. Nat Commun $2018 ; 9$ : 3561.

9. Elmore MRP, Najafi AR, Koike MA, et al. Colonystimulating factor 1 receptor signaling is necessary for microglia viability, unmasking a microglia progenitor cell in the adult brain. Neuron $2014 ; 82$ $380-97$

10. Lamb J, Crawford ED, Peck D, et al. The connectivity map: using gene-expression signatures to connect small molecules, genes, and disease. Science 2006 313: 1929-35.

11. Delahaye-Duriez A, Srivastava P, Shkura K, et al. Rare and common epilepsies converge on a shared gene regulatory network providing opportunities for novel antiepileptic drug discovery. Genome Biol 2016; 17 : 245

12. Subramanian A, Narayan R, Corsello SM, et al. A next generation connectivity map: L1000 platform and the first 1,000,000 profiles. Cell 2017 ; 171 : 1437-52.el7.

13. Keenan AB, Jenkins SL, Jagodnik KM, et al. The library of integrated network-based cellular signatures $\mathrm{NIH}$ program: system-level cataloging of human cells response to perturbations. Cell Syst $2018 ; 6: 13-24$.

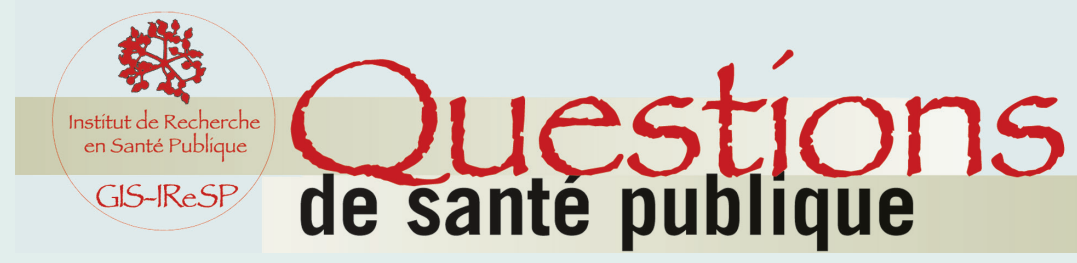

\section{Un nouveau bulletin}

\section{pour une meilleure}

visibilité des résultats

de la recherche

en santé publique

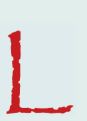

es résultats de la recherche en santé publique souffrent en France d'un réel manque de visibilité. Ceci concerne aussi bien le monde académique (hors santé publique) que le grand public et les décideurs. Pour pallier ce déficit, l'IReSP a créé un bulletin à large diffusion intitulé "Questions de santé publique ", largement inspiré du bulletin mensuel d'information de I'INED " Populations et sociétés ". L'objectif éditorial est de porter à la connaissance d'un large public (enseignants, étudiants, journalistes, décideurs, milieux de la recherche, associations,

public concerné) les informations les plus récentes concernant des questions importantes de santé publique, rédigées de façon facilement lisible et compréhensible pour des non spécialistes, en garantissant que les informations publiées sont validées scientifiquement. La publication concerne des faits et non des positions. Audelà de la présentation de résultats, les qualités pédagogiques de Questions de santé publique permettent au lecteur de mieux comprendre comment sont formulées et abordées les questions de santé publique et quelles sont les limites de ces études.

Nom

Prénom

Institution $\ldots \ldots \ldots \ldots$ Fonction

Spécialité

Service

Adresse

Ville

Code postal

Pays

Adresse électronique

à nous retourner par la poste ou par fax au 0149850345

Questions de santé publique

EDP Sciences

17 avenue du Hoggar

91944 Les Ulis

France

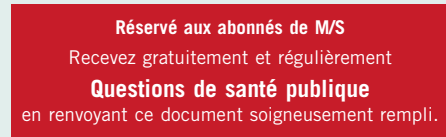

Questions de santé publique est une publication de I'Institut de Recherche en Santé Publique. I Directeur de la publication : Corinne Alberti. I Rédactrice en chef : Kodja Yetongnon. I Comité de relecture : Lorraine Cousin, Jean-Marie Gagliolo, Coline Terroba. I Réalisation : EDP Sciences. 